\title{
Structure de certains produits semi directs
}

\author{
A. LAMOTTE \\ Laboratoire de Probabilités, Associé au CNRS, $n^{\circ}$ 224, 4, Place Jussieu, Tour 56-66, \\ 3ème étage, 75005 Paris, France
}

(Received 30 March 1982)

\begin{abstract}
We consider the skew product of a dynamical system with a Bernoulli flow and we prove that under additional conditions this skew product is isomorphic to a direct product. We use this result to show that the minimal diffeomorphism with strictly positive entropy (constructed by M. R. Herman) is isomorphic to a direct product of a 0-entropy dynamical system with a Bernoulli shift.
\end{abstract}

\section{Introduction}

On considère le produit semi direct d'un système dynamique par un flot de Bernoulli et on donne des conditions suffisantes pour qu'un tel produit semi direct se décompose en un produit direct. On applique ensuite ce résultat pour montrer que l'exemple d'un difféomorphisme minimal d'entropie non nulle (construit par M. R. Herman) est isomorphe au produit direct d'un système dynamique ergodique d'entropie nulle par un schéma de Bernoulli.

\section{Produit semi direct}

La notation $(X, \mathscr{A}, m)$ d'espace de probabilité est condensée en $X$ et tous les espaces utilisés sont des espaces de Lebesgue.

Définition. Soit $T$ un automorphisme sur un espace $X$ et soit $\phi$ une fonction mesurable sur $X$. Soit $\left(S_{t}\right)$ un flot mesurable sur un espace $Y$. On appelle produit semi direct du système $(X, T)$ par le flot $\left(Y, S_{t}\right)$ pour la fonction $\phi$, le système dynamique $\left(X_{0}, T_{0}\right)$ défini par $X_{0}=X \times Y$ et par $T_{0}(x, y)=\left[T x, S_{\phi(x)} y\right]$.

THÉOREME 1. Soit $(X, T)$ un système dynamique ergodique entropie finie et soit $\phi$ une fonction intégrable sur $X$ vérifiant $0<\int_{X} \phi d m<+\infty$. Soit $\left(S_{t}\right)$ un flot de Bernoulli d'entropie finie sur un espace $Y$. Le produit semi direct $\left(X_{0}, T_{0}\right)$ du système $(X, T)$ par le flot $\left(Y, S_{t}\right)$ pour la fonction $\phi$ est alors isomorphe au produit direct du système $(X, T)$ par un schéma de Bernoulli.

Les propriétés suivantes, dont les références par rapport à la bibliographie sont indiquées entre crochets seront utilisées pour démontrer le théorème 1 .

Proposition 1 (conséquence du théorème ergodique). Soit $(X, T)$ un système dynamique ergodique et soit $\phi$ une fonction réelle intégrable sur $X$ dont l'intégrale est 
finie. On note

$$
\Phi(i, x)=\sum_{j=0}^{i-1} \phi\left(T^{j} x\right) \quad \text { pour } i \text { entier positif }
$$

et

$$
\Phi(i, x)=-\sum_{j=i}^{-1} \phi\left(T^{j} x\right) \quad \text { pour } i \text { entier négatif. }
$$

Pour $\varepsilon>0$, il existe alors un entier $N$ et une partie $X_{1}$ de $X$ telle que mes $\left(X_{1}\right)>1-\varepsilon$ et telle que si $x \in X_{1}$ et si $|i|>N$ avec $i \in \mathbb{Z}$ alors

$$
\left|(1 / i) \Phi(i, x)-\int_{X} \phi d m\right|<\varepsilon .
$$

Proposition 2 ([7] et [8]). Soit $\left(X_{0}, T_{0}\right)$ un système dynamique ergodique et soit $P$ une partition sur $X_{0}$. Pour une partition $Q$ sur $X_{0}$, on dit que le système $\left(Q, T_{0}\right)$ est très faiblement Bernoulli conditionnellement à $P$ et on note $\left(Q, T_{0}\right) t-f-b-/ P$ lorsque pour tout $\varepsilon>0$ il existe $N$ tel que pour $n$ et $m$ entiers vérifiant $n>N$ et $m>0$ il existe $K$ tel que si $k>K$ alors pour $\varepsilon$ presque tout atome $\pi \cap p$ de $\bigvee_{-m}^{0} T_{0}^{i} Q \vee \bigvee_{-k}^{k} T_{0}^{i} P$ on $a$

$$
d\left[\bigvee_{1}^{n} T_{0}^{i} Q / \pi \cap p ; \bigvee_{1}^{n} T_{0}^{i} Q / p\right]<\varepsilon
$$

$S$ 'il existe une suite croissante $\left(Q_{m}\right)_{m \in \mathbb{N}}$ de partitions sur $X_{0}$ vérifiant $\left(Q_{m}, T_{0}\right) t-f-$ $b-/ P$ pour tout $m$ et vérifiant

$$
\bigvee_{m \in \mathbb{N}} \bigvee_{i \in \mathbb{Z}} T_{0}^{i}\left(Q_{m} \vee P\right)=X_{0}
$$

(tribu initiale de l'espace $X_{0}$ ) alors il existe une partition $Q$ sur $X_{0}$ telle que les tribus $\bigvee_{-\infty}^{+\infty} T_{0}^{i} P$ et $\bigvee_{-\infty}^{+\infty} T_{0}^{i} Q$ soient indépendantes, telle que

$$
\bigvee_{-\infty}^{+\infty} T_{0}^{i}(P \vee Q)=X_{0}
$$

et telle que tous les $\left(T_{0}^{i} Q\right)_{i \in \mathbb{Z}}$ soient indépendantes. Ces conditions assurent que le système dynamique $\left(X_{0}, T_{0}\right)$ est isomorphe au produit direct du facteur $\left[X_{0} ; V_{-\infty}^{+\infty} T_{0}^{i} P ; T_{0}\right]$ par un schéma de Bernoulli.

Proposition 3 ([1, p. 219], théorème de renouvellement). Soit $\left(X_{i}\right)_{i \in \mathbb{N}}$ une suite de variables aléatoires indépendantes et équidistribuées définies sur un espace de probabilité $\Omega$ et à valeurs dans une partie finie de $\mathbb{R}^{+}$. Pour $j$ entier et $\omega$ dans $\Omega$ on définit

$$
\sigma_{j}(\omega)=\sum_{i=1}^{j-1} X_{i}(\omega)
$$

et pour un intervalle I de $\mathbb{R}$ on définit la variable aléatoire $N(I)$ sur $\Omega$ par

$$
N(I)(\omega)=\text { Nombre d'entiers } j \text { tels que } \sigma_{j}(\omega) \in I .
$$

Si I désigne un intervalle fixé de $\mathbb{R}, E[N(I+z)]$ tend vers (longueur $I) / E\left(X_{1}\right)$ lorsque $z$ réel tend vers $+\infty$ où $E(-)$ désigne l'espérance de la variable aléatoire (-). 
Démonstration du théorème 1 . En reprenant la notation $\Phi(i, x)$ définie dans la proposition 1 on obtient

$$
T_{0}^{i}(x, y)=\left[T^{i} x, S_{\Phi(i, x)} y\right] \quad \text { pour tout } i \text { dans } \mathbb{Z} .
$$

(1) Ergodicité du système $\left(X_{0}, T_{0}\right)$.

Soit $A$ et $A^{\prime}$ deux parties mesurables sur $\mathrm{X}$ et soit $\mathrm{B}$ et $\mathrm{B}^{\prime}$ deux parties mesurables sur $Y$. Pour obtenir l'ergodicité du système $\left(X_{0}, T_{0}\right)$ il suffit de montrer que

$$
(1 / n) \sum_{k=1}^{n} \operatorname{mes}\left[(A \times B) \cap T_{0}^{k}\left(A^{\prime} \times B^{\prime}\right)\right] \rightarrow \operatorname{mes}(A \times B) \cdot \operatorname{mes}\left(A^{\prime} \times B^{\prime}\right)
$$

lorsque $n \rightarrow \infty$.

Soit $\varepsilon>0$. Le flot $\left(S_{t}\right)$ étant mélangeant sur $Y$, il existe un entier $m_{0}$ tel que si $\tau>m_{0}$ avec $\tau$ réel alors $\mid$ mes $\left(B \cap S_{\tau} B^{\prime}\right)-$ mes $B \times$ mes $B^{\prime} \mid$ est inférieur à $\varepsilon / 10$. D'après la proposition 1 , il existe $k_{1}$ entier tel que pour $\varepsilon / 10$ presque tout $x$ de $X$ on ait $\Phi(k, x)>m_{0}$ dès que $k>k_{1}$. En écrivant

$$
\operatorname{mes}\left[(A \times B) \cap T_{0}^{k}\left(A^{\prime} \times B^{\prime}\right)\right]=\int_{X} \mathbb{1}_{A \cap T^{k} A^{\prime}}(x) \times \operatorname{mes}\left[B \cap S_{\Phi(k, x)} B^{\prime}\right] d m(x)
$$

on obtient

$$
\left|\operatorname{mes}\left[(A \times B) \cap T_{0}^{k}\left(A^{\prime} \times B^{\prime}\right)\right]-\operatorname{mes} B \times \operatorname{mes} B^{\prime} \times \operatorname{mes}\left(A \cap T^{k} A^{\prime}\right)\right|<\frac{3}{10} \varepsilon
$$

dès que $k>k_{1}$. D'après l'ergodicité du système $(X, T),(1 / n) \sum_{k=1}^{n} \operatorname{mes}\left(A \cap T^{k} A^{\prime}\right)$ tend vers mes $A \times$ mes $A^{\prime}$ lorsque $n$ tend vers $+\infty$ et on obtient alors le résultat.

(2) Modèle du flot $\left(Y, S_{t}\right)$.

D'après le théorème d'isomorphisme des flots de Bernoulli d'entropie finie et d'après le résultat de D. S. Ornstein [5], on peut prendre comme modèle pour notre flot de Bernoulli ( $Y, S_{t}$ ) le flot obtenu de la façon suivante:

Soit $(\bar{Y}, \bar{T})$ un système dynamique avec $\bar{Q}=[\bar{Q}(0) ; \bar{Q}(1)]$ partition sur $\bar{Y}$ telle que mes $\bar{Q}(0)=$ mes $\bar{Q}(1)=\frac{1}{2}$, telle que tous les $\left(\bar{T}^{i} \bar{Q}\right)_{i \in Z}$ soient indépendants et telle que $\bigvee_{-\infty}^{+\infty} \bar{T}^{i} \bar{Q}=\bar{Y}$.

Soit $\alpha$ et $\beta$ deux réels tels que $0<\alpha<\beta$ avec $\alpha / \beta \notin \mathbb{Q}$ et avec

$$
(\alpha+\beta) / 2=(\log 2) / h_{0}(S)
$$

où $h_{0}(S)$ désigne l'entropie du flot initial $\left(S_{t}\right)$ sur $Y$. On note $\delta=(\alpha+\beta) / 2$.

On définit $Y=\bar{Q}(0) \times\left[0, \alpha\left[\cup \bar{Q}(1) \times\left[0, \beta\left[\right.\right.\right.\right.$ et on définit $\left(S_{t}\right)$ sur $Y$ comme étant le flot construit au dessus du système $(\bar{Y}, \bar{T})$ pour la fonction valant $\alpha$ sur $\bar{Q}(0)$ et $\beta$ sur $\bar{Q}(1)$. [Sous l'action de ce flot $\left(S_{t}\right)$, le point $y=(\bar{y}, \tau)$ de $Y$ se déplace sur la verticale au dessus de $(\bar{y}, \tau)$ jusqu'à ce qu'il rencontre le point du graphe de la fonction précédente et se déplace ensuite sur la verticale au dessus de $\left(\bar{T}_{\bar{y}}, 0\right)$, les déplacements s'effectuant avec une vitesse unitaire.]

On considère sur $Y$ la partition $Q=[Q(0) ; Q(1)]$ définie par $Q(0)=\bar{Q}(0) \times[0, \alpha[$ et $Q(1)=\bar{Q}(1) \times\left[0, \beta\left[\right.\right.$. D'après [5], pour $\gamma$ suffisamment petit on a $\bigvee_{i \in Z}^{-} S_{i \gamma} Q=Y$. Après avoir fixé un tel $\gamma$, on pose

$$
Q_{m}=\bigvee_{i=-m}^{+m} S_{i \gamma} Q
$$

pour tout $m$ de $\mathbb{N}$. 
(3) Propriété de produit direct.

Soit $P$ une partition sur $X$ telle que $\bigvee_{-\infty}^{+\infty} T^{i} P=X$. D'après la proposition 2 , il suffit, pour obtenir le résultat du théorème 1 , de démontrer que pour tout $m$ entier le système $\left(Q_{m}, T_{0}\right)$ est $\mathrm{t}-\mathrm{f}-\mathrm{b}-/ P$. Dans la suite on démontre que $\left(Q, T_{0}\right)$ est $\mathrm{t}-\mathrm{f}-\mathrm{b}-/ P$ et le cas général s'obtient de façon tout à fait analogue.

Soit $\varepsilon>0$. La partition $P$ étant génératrice sur $X$ il suffit, pour obtenir la propriété $\left(Q, T_{0}\right) \mathrm{t}-\mathrm{f}-\mathrm{b}-/ P$ de démontrer qu'il existe $N_{0}$ tel que si $n>N_{0}$ et si $m>0$ alors $\varepsilon^{2} / 100$ presque tout $x$ de $X$ vérifie: pour $\varepsilon^{2} / 10$ presque tout atome $\pi_{x}$ de $\bigvee_{-m}^{0} S_{\Phi(i, x)} Q$ on a

$$
d\left[\bigvee_{i=1}^{n} S_{\Phi(i, x)} Q / \pi_{x} ; \bigvee_{i=1}^{n} S_{\Phi(i, x)} Q\right]<\varepsilon / 10
$$

D'après la proposition 1 , il existe $n_{0}$ et $m_{0}$ entiers tels que $\varepsilon^{2} / 1000$ presque tout $x$ de $X$ vérifie la condition (1) suivante:

$$
\text { si } i>n_{0} \text { et si } j>m_{0} \text { alors } \Phi(i, x)>2 \beta \text { et } \Phi(-j, x)<0 .
$$

Si $x$ vérifie cette dernière condition tout atome de $V_{-m}^{-m_{0}} S_{\Phi(i, x)} Q$ est une réunion de rectangles sur $Y$ dont les bases sur $\bar{Y}$ sont mesurables pour $\bigvee_{-\infty}^{0} \bar{T}^{i} \bar{Q}$. Il suffit donc de trouver $N_{2}$ tel que si $n>N_{2}$ alors $\varepsilon^{2} / 200$ presque tout $x$ de $X$ vérifiant (1) est tel que:

$$
\varpi\left[\bigvee_{n_{0}}^{n} S_{\Phi(i, x)} Q / R_{1} ; \bigvee_{n_{0}}^{n} S_{\Phi(i, x)} Q / R_{2}\right]<\varepsilon / 10
$$

pour tous rectangles $R_{1}$ et $R_{2}$ sur $Y$ dont les bases sur $\bar{Y}$ sont mesurables pour $\bigvee_{-\infty}^{0} \bar{T}^{i} \bar{Q}$.

1 ère étape: les rectangles sont inclus dans une même bande horizontale de $Y$ de hauteur inférieure à $\varepsilon \delta / 60$.

On considère les variables aléatoires $\left(X_{i}\right)_{i \in \mathbb{N}}$ définies sur $\bar{Y}$ par

$$
\begin{array}{ll}
X_{i}(\bar{y})=\alpha & \text { si } \bar{T}^{-i} \bar{y} \in \bar{Q}(0) \\
X_{i}(\bar{y})=\beta & \text { si } \bar{T}^{-i} \bar{y} \in \bar{Q}(1) .
\end{array}
$$

Les variables aléatoires $\left(X_{i}\right)_{i \in N}$ sont indépendantes et équidistribuées. En reprenant les résultats de la proposition 3 , on peut définir la variable aléatoire $N([z, z+\varepsilon \delta / 60[)$ et il existe $A_{1}>0$ tel que si $z>A_{1}$ alors

$$
E[N([z, z+\varepsilon \delta / 60[)]<\varepsilon / 50 .
$$

D'après la proposition 1 , il existe $B_{1}>0$ tel que $\varepsilon^{2} / 1000$ presque tout $x$ de $X$ vérifie la condition (2) suivante:

$$
\text { si } i>B_{1} \text { alors } \Phi(i, x)>A_{1}+\beta \text {. }
$$

Soit $N_{1}$ entier tel que si $n>N_{1}$ alors $\sup \left(n_{0}, B_{1}\right) / n<\varepsilon / 100$.

Pour $j=1,2$ soit $R_{j}=\bar{a}_{j} \times\left[\mu_{j}, \nu_{j}\right.$ [ un rectangle sur $Y$ dont la base $\bar{a}_{j}$ sur $\bar{Y}$ est mesurable pour $\bigvee_{-\infty}^{0} \bar{T}^{i} \bar{Q}$ et on suppose que $R_{1}$ et $R_{2}$ sont inclus dans une même bande horizontale de hauteur $<\varepsilon \delta / 60$ [c'est à dire $\sup \left(\left|\nu_{1}-\mu_{2}\right| ;\left|\nu_{2}-\mu_{1}\right|\right)<\varepsilon \delta / 60$ ]. Les $\left(\bar{T}^{i} \bar{Q}\right)_{i \in \mathbf{Z}}$ étant indépendants, si $x$ vérifie la condition (1) alors pour $j=1,2$ les systèmes $\left(\bigvee_{n_{0}}^{n} S_{\Phi(i, x)} Q / \bar{a}_{j} \times\left\{\mu_{j}\right\}\right)$ et $\left(\bigvee_{n_{0}}^{n} S_{\Phi(i, x)} Q / \bar{Y} \times\{0\}\right)$ sont isomorphes. 
Pour $\bar{y}$ dans $\bar{Y}$ et $t$ réel on note $S_{t} Q(\bar{y})$ l'indice de l'atome de $Q$ contenant $S_{-t}(\bar{y}, 0)$. Si $S_{t_{1}} Q(\bar{y}) \neq S_{t_{2}} Q(\bar{y})$ avec $0<t_{1}<t_{2}$ alors, en reprenant les notations données dans la proposition 3 on a $N\left(\left[t_{1}, t_{2}[)(\bar{y})=1\right.\right.$. Si $i>B_{1}$ et si $x$ vérifie les conditions (1) et (2) on obtient ainsi:

$$
\operatorname{mes}\left\{\bar{y} \in \bar{Y} ; S_{\Phi(i, x)-\mu_{1}} Q(\bar{y}) \neq S_{\Phi(i, x)-\mu_{2}} Q(\bar{y})\right\}<\varepsilon / 50
$$

et

$$
\operatorname{mes}\left\{\bar{y} \in \bar{a}_{j} ; S_{\Phi(i, x)-\mu_{j}} Q(\bar{y}) \neq S_{\Phi(i, x)-y} Q(\bar{y})\right\}<(\varepsilon / 50) \times \text { mes } \bar{a}_{j}
$$

dès que $y \in\left[\mu_{j}, \nu_{j}\left[\right.\right.$ pour $j=1,2$. Pour $x$ vérifiant (1) et (2) et pour $n>N_{1}$ on obtient ainsi:

$$
d\left[\bigvee_{n_{0}}^{n} S_{\Phi(i, x)} Q / R_{1} ; \bigvee_{n_{0}}^{n} S_{\Phi(i, x)} Q / R_{2}\right]<\frac{4}{50} \varepsilon
$$

2ème étape: les rectangles sont quelconques.

On considère une suite strictement croissante $\left(\varepsilon_{l}\right)_{l=0}^{l_{1}^{1}}$ vérifiant $\varepsilon_{0}=0, \varepsilon_{l_{1}}=\beta, \varepsilon_{b_{0}}=\alpha$ pour un $l_{0}$ et vérifiant $\left|\varepsilon_{l}-\varepsilon_{l-1}\right|<\varepsilon \delta / 60$ pour tout $l=1, \ldots, l_{1}$. Pour $l=1, \ldots, l_{0}$ on pose $Y(l)=\bar{Y} \times\left[\varepsilon_{l-1}, \varepsilon_{l}\left[\right.\right.$ et pour $l=l_{0}+1, \ldots, l_{1}$ on pose $Y(l)=\bar{Q}(1) \times\left[\varepsilon_{l-1}, \varepsilon_{l}[\right.$. On considère la suite $\left(X_{i}\right)_{i \in N}$ de variables aléatoires sur $\bar{Y}$ définie précédemment et, en utilisant la proposition 3 on choisit $C>0$ tel que si $z>C-\beta$ alors

$$
\mid E\left[N\left(\left[z+\varepsilon_{l-1}, z+\varepsilon_{l}\right)\right]-\frac{\left(\varepsilon_{l}-\varepsilon_{l-1}\right)}{\delta} \mid<\frac{\varepsilon \times\left(\varepsilon_{l}-\varepsilon_{l-1}\right)}{100 \times l_{1} \times \delta}\right.
$$

pour tout $l=1, \ldots, l_{1}$. Soit $A_{1}$ défini dans l'étude de la première étape et soit $B_{2}$ tel que $\varepsilon^{2} / 1000$ presque tout $x$ de $X$ vérifie la condition (3) suivante:

$$
\text { si } i>B_{2} \text { alors } \Phi(i, x)-C>A_{1} \text {. }
$$

Soit $N_{2}$ tel que $\sup \left(n_{0}, B_{2}\right) / N_{2}<\varepsilon / 100$. Soit $R_{1}$ et $R_{2}$ deux rectangles sur $Y$ dont les bases sur $\bar{Y}$ sont mesurables pour $\bigvee_{-\infty}^{0} \bar{T}^{i} \bar{Q}$. Pour $j=1,2$ et pour $l=1, \ldots, l_{1}$ soit $R_{j}(l)=\left\{y \in R_{j} ; S_{-C} y \in Y(l)\right\}$. Lorsque $l \leq l_{0} ; y=(\bar{y}, \eta) \in R_{j}(l)$ si et seulement si $N\left(\left[C-\eta+\varepsilon_{l-1}, C-\eta+\varepsilon_{l}[)(\bar{y})\right.\right.$ vaut 1 . Lorsque $l>l_{0} ; y=(\bar{y}, \eta) \in R_{j}(l)$ si et seulement si $N\left(\left[C-\eta+\varepsilon_{l-1}, C-\eta+\varepsilon_{l}[)(\bar{y})=1\right.\right.$ et $N([C-\eta, C-\eta+\alpha[)(\bar{y})=0$. Les bases des rectangles étant mesurables pour $\bigvee_{-\infty}^{0} \bar{T}^{i} \bar{Q}$, d'après le choix de $C$ on obtient

$$
\mid\left[\text { mes } R_{1}(l) / \text { mes } R_{1}\right]-\left[\operatorname{mes} R_{2}(l) / \text { mes } R_{2}\right] \mid<\varepsilon / 100 \quad \text { pour } l=1, \ldots, l_{1} \text {. }
$$

Soit $x$ vérifiant les conditions (1) et (3) et soit $n>N_{2} . S_{-c} R_{j}(l)$ étant une réunion de rectangles inclus dans $Y(l)$, on obtient d'après la première étape:

$$
d\left[\bigvee_{n_{0}}^{n} S_{\Phi(i, x)-C} Q / S_{-C} R_{1}(l) ; \bigvee_{n_{0}}^{n} S_{\Phi(i, x)-C} Q / S_{-C} R_{2}(l)\right]<\frac{4}{50} \varepsilon
$$

D'après la propriété sur les mesures relatives de $R_{1}(l)$ et $R_{2}(l)$ on obtient alors:

$$
d\left[\bigvee_{n_{0}}^{n} S_{\Phi(i, x)-C} Q / S_{-C} R_{1} ; \bigvee_{n_{0}}^{n} S_{\Phi(i, x)-C} Q / S_{-C} R_{2}\right]<\varepsilon / 10
$$

ce qui assure le résultat. 
Remarques. Le théorème 1 généralise un résultat établi par M. Brin, prop (2-3) dans [2].

Produit semi direct de deux flots: Soit $\left(T_{t}\right)$ un flot ergodique sur un espace $X$ et soit $\phi$ une fonction intégrable de $X$ dans $\mathbb{R}$, dont l'intégrale est strictement positive. Soit $\left(S_{t}\right)$ un flot de Bernoulli sur un espace $Y$. Pour $x$ dans $X$ et $t$ réel on pose

$$
\Phi(t, x)=\int_{0}^{t} \phi\left(T_{\tau} x\right) d \tau
$$

En utilisant le théorème 1 il est possible de démontrer que le flot $\left(R_{t}\right)$ défini sur $X \times Y$ par $R_{t}(x, y)=\left[T_{t} x, S_{\Phi(t, x)} y\right]$ est isomorphe au produit direct du flot $\left(X, T_{t}\right)$ par un flot de Bernoulli.

Structure de la transformation de $M$. R. Herman

On considère la transformation $F_{\alpha}$ utilisée dans [4] et définie sur $\mathbb{T}_{1} \times \operatorname{SL}(2, \mathbb{R}) / \Gamma$ par $F_{\alpha}(\theta, y)=[\theta+\alpha ; A(\theta) y]$ avec $\mathbb{T}_{1}=\mathbb{R} / \mathbb{Z}$, avec $\Gamma$ sous groupe discret à quotient compact de SL $(2, \mathbb{R})$ et avec

$$
A(\theta)=\left[\begin{array}{cc}
\cos 2 \pi \theta & -\sin 2 \pi \theta \\
\sin 2 \pi \theta & \cos 2 \pi \theta
\end{array}\right] \times\left[\begin{array}{cc}
\lambda & 0 \\
0 & 1 / \lambda
\end{array}\right] \quad \text { où } \lambda>1 \text { est fixé. }
$$

Soit $\nu$ l'unique mesure de probabilité sur $\operatorname{SL}(2, \mathbb{R}) / \Gamma$ invariante par l'action à gauche de $\operatorname{SL}(2, \mathbb{R})$ et soit $M=\mathbb{T}_{1} \times \operatorname{SL}(2, \mathbb{R}) / \Gamma$ muni de la mesure $\mu=d \theta \times \nu$.

ThÉORÈme 2. Pour $\alpha \in \mathbb{T}_{1} \backslash(\mathbb{Q} / \mathbb{Z})$ le système $\left(M, \mu, F_{\alpha}\right)$ est isomorphe au produit direct d'un système ergodique d'entropie nulle par un schéma de Bernoulli.

Notations. On note $I$ la matrice unité dans $\operatorname{SL}(2, \mathbb{R})$. Pour $\alpha \in \mathbb{T}_{1}$, on pose

$$
A_{\alpha}^{n}(\theta)=A[\theta+(n-1) \alpha] \times \cdots \times A(\theta+\alpha) \times A(\theta)
$$

pour $n$ entier positif et

$$
A_{\alpha}^{n}(\theta)=A[\theta+n \alpha]^{-1} \times \cdots \times A[\theta-\alpha]^{-1}
$$

pour $n<0$ et on note $R_{\alpha}$ la transformation définie sur $\mathbb{T}_{1} \operatorname{par} R_{\alpha}(\theta) \equiv \theta+\alpha \bmod 1$.

LeMme 4. Pour $\alpha \in \mathbb{T}_{1} \backslash(\mathbb{Q} / \mathbb{Z})$, il existe une partie $\mathbb{T}_{1}^{\prime}$ de $\mathbb{T}_{1}$ invariante par $R_{\alpha}$, de mesure 1 et il existe une application $Q$ mesurable de $\mathbb{T}_{1}^{\prime}$ dans $\operatorname{SL}(2, \mathbb{R})$ et une application $\phi$ mesurable de $\mathbb{T}_{1}$ dans $\mathbb{R}^{*}$ telles que:

(i) la fonction $\log |\phi(\cdot)|$ est intégrable sur $\mathbb{T}_{1}^{\prime}$, d'intégrale strictement positive;

(ii) si $\theta \in \mathbb{T}_{1}^{\prime}, A(\theta)=Q(\theta+\alpha) B(\theta) Q(\theta)^{-1}$ où

$$
B(\theta)=\left[\begin{array}{cc}
\phi(\theta) & 0 \\
0 & 1 / \phi(\theta)
\end{array}\right] .
$$

Démonstration du lemme 4. $\|A(\theta)\|=\lambda$ donc, d'après le théorème ergodique sous additif, pour presque tout $\theta$ de $\mathbb{T}_{1},(1 / n) \log \left\|A_{\alpha}^{n}(\theta)\right\|$ converge lorsque $n$ tend vers l'infini. Cette limite, notée ici $\lambda(\alpha)$, est constante et, d'après le théorème 3.1 donné dans [4], elle vérifie $\lambda(\alpha)>0$. En appliquant le théorème ergodique multiplicatif donné dans [6], on obtient l'existence de deux applications mesurables $\Lambda_{+}$et $\Lambda_{-}$, définies sur une partie $\mathbb{T}_{1}^{\prime}$ de $\mathbb{T}_{1}$ de mesure 1 et invariante par $R_{\alpha}$, à valeurs dans 
$\operatorname{SL}(2, \mathbb{R})$ et telles que si $\theta \in \mathbb{T}_{1}^{\prime}$ alors:

$\left[{ }^{t} A_{\alpha}^{n}(\theta) A_{\alpha}^{n}(\theta)\right]^{1 / 2 n} \rightarrow \Lambda_{+}(\theta) \quad$ lorsque $n \rightarrow+\infty$.

$\left.{ }^{t} A_{\alpha}^{n}(\theta) A_{\alpha}^{n}(\theta)\right]^{-1 / 2 n} \rightarrow \Lambda_{-}(\theta) \quad$ lorsque $n \rightarrow-\infty$.

$\Lambda_{+}(\theta)$ et $\Lambda_{-}(\theta)$ possèdent les mêmes valeurs propres qui sont $\exp \lambda(\alpha)$ et $\exp -\lambda(\alpha)$.

si $U_{-}(\theta)\left[\right.$ resp. $U_{+}(\theta)$ ] est le sous espace propre de $\Lambda_{-}(\theta)$ [resp. de $\Lambda_{+}(\theta)$ ] associé à $\exp -\lambda(\alpha)$ alors

$$
\mathbb{R}^{2}=U_{-}(\theta) \oplus U_{+}(\theta), \quad A(\theta) U_{-}(\theta)=U_{-}(\theta+\alpha), \quad A(\theta) U_{+}(\theta)=U_{+}(\theta+\alpha)
$$

et pour tout $u$ de $U_{-}(\theta)$ on a

$$
(1 / n) \log \left\|A_{\alpha}^{n}(\theta) u\right\| \rightarrow \lambda(\alpha) \quad \text { lorsque } n \rightarrow \pm \infty .
$$

Si le premier vecteur colonne de la matrice adjointe de $\Lambda_{-}(\theta)-[\exp -\lambda(\alpha)] I$ est non nul, on le note $u_{-}(\theta)$ et s'il est nul on note $u_{-}(\theta)$ le deuxième vecteur colonne de cette même matrice. On définit de même $u_{+}(\theta)$ en utilisant la matrice $\Lambda_{+}(\theta)-$ $[\exp -\lambda(\alpha)] I$. Soit

$$
v_{+}(\theta)=u_{+}(\theta) / \operatorname{det}\left[v_{-}(\theta), u_{+}(\theta)\right]
$$

où $v_{-}(\theta)=u-(\theta) /\left\|u_{-}(\theta)\right\|$.

Soit $\phi$ définie de $\mathbb{T}_{1}^{\prime}$ dans $\mathbb{R}^{*}$ par

$$
A(\theta) v_{-}(\theta)=\phi(\theta) v_{-}(\theta+\alpha)
$$

et soit $Q(\theta)$ la matrice dont le premier vecteur colonne est $v_{-}(\theta)$ et dont le deuxième vecteur colonne est $v_{+}(\theta)$. Les applications $\phi$ et $Q$ sont mesurables sur $\tau_{1}^{\prime}$ et si on note $B(\theta)$ la matrice

$$
\left[\begin{array}{cc}
\phi(\theta) & 0 \\
0 & 1 / \phi(\theta)
\end{array}\right]
$$

on obtient $A(\theta) Q(\theta)=Q(\theta+\alpha) B(\theta)$ ce qui assure la deuxième propriété donnée dans le lemme 4. $v_{-}(\theta)$ et $v_{-}(\theta+\alpha)$ étant de norme 1 , on obtient $|\phi(\theta)|<\|A(\theta)\|=\lambda$ et $|1 / \phi(\theta)|<\left\|A(\theta)^{-1}\right\|=\lambda$ donc l'application $\log |\phi(\cdot)|$ est intégrable sur $\bar{T}_{1}^{\prime} \cdot A_{\alpha}^{n}(\theta) v_{-}(\theta)$ est égal à

$$
\phi[\theta+(n-1) \alpha] \times \cdots \times \phi(\theta) v_{-}(\theta+n \alpha)
$$

et

$$
(1 / n) \log \left\|A_{\alpha}^{n}(\theta) v_{-}(\theta)\right\| \rightarrow \lambda(\alpha) \quad \text { lorsque } n \rightarrow+\infty
$$

donc d'après le théorème ergodique on obtient $\int_{\mathrm{T}_{1}^{\prime}} \log |\phi(\theta)| d \theta=\lambda(\alpha)>0$ ce qui assure la première propriété donnée dans le lemme 4 .

Pour obtenir le théorème 2 on utilisera également la résultat suivant extrait de [3] et donné ici sans démonstration.

Proposition 5. Soit $(X, T)$ un système dynamique ergodique. Une extension à deux points d'un produit direct de $(X, T)$ par un schéma de Bernoulli est alors;

(i) soit isomorphe au produit direct de $(X, T)$ par un schéma de Bernoulli;

(ii) soit isomorphe au produit direct d'une extension à deux points du système $(X, T)$ par un schéma de Bernoulli. 
Démonstration du théorème 2 . On reprend les notations données dans le lemme 4. (1) Supposons $(-I) \in \Gamma$. On pose

$$
B^{\prime}(\theta)=\left[\begin{array}{cc}
|\phi(\theta)| & 0 \\
0 & |1 / \phi(\theta)|
\end{array}\right]
$$

et on considère la transformation $F_{\alpha}^{\prime}$ définie sur $M$ par

$$
F_{\alpha}^{\prime}(\theta, y)=\left[\theta+\alpha, B^{\prime}(\theta) y\right] .
$$

D'après le lemme 4, en utilisant l'isomorphisme $(\theta, y) \rightarrow\left[\theta, Q(\theta)^{-1} y\right]$, les transformations $F_{\alpha}$ et $F_{\alpha}^{\prime}$ sur $(M, \mu)$ sont isomorphes. D'après [5], le flot $\left(S_{t}\right)$ défini sur $\mathrm{SL}^{\prime}(2, \mathbb{R}) / \Gamma$ par

$$
S_{t} y=\left[\begin{array}{cc}
e^{t} & 0 \\
0 & e^{-t}
\end{array}\right] y
$$

est un flot de Bernoulli. En utilisant le lemme 4 et en écrivant

$$
F_{\alpha}^{\prime}(\theta, y)=\left[R_{\alpha}(\theta), S_{\log |\phi(\theta)|} y\right]
$$

le résultat du théorème 2 devient une conséquence du théorème 1 .

(2) Supposons $(-I) \notin \Gamma$. Soit $\Gamma^{\prime}=\Gamma \cup(-\Gamma)$ et soit $M^{\prime}=\mathbb{\nabla}_{1}^{\prime} \times \operatorname{SL}(2, \mathbb{R}) / \Gamma^{\prime}$. En reprenant l'expression $B^{\prime}(\theta)$ définie dans (1), on définit une transformation $F_{\alpha}^{\prime \prime}$ sur $M^{\prime}$ par

$$
F_{\alpha}^{\prime \prime}\left(\theta, y^{\prime}\right)=\left[\theta+\alpha, B^{\prime}(\theta) y^{\prime}\right] .
$$

Le système $\left(M, \mu, F_{\alpha}\right)$ est alors une extension à deux points du système $\left(M^{\prime}, \mu^{\prime}, F_{\alpha}^{\prime \prime}\right)$ et en reprenant ce qui a été fait dans la partie (1) on obtient que $\left(M^{\prime}, \mu^{\prime}, F_{\alpha}^{\prime \prime}\right)$ est isomorphe au produit direct de $\left(\pi_{1}, R_{\alpha}\right)$ par un schéma de Bernoulli. Le résultat du théorème 2 est alors une conséquence de la proposition 5 .

\section{REFERENCES}

[1] L. Breiman. Probability. Addison Wesley: Reading, Mass. (1968).

[2] M. Brin. Bernoulli diffeomorphisms with $n-1$ non zero exponents. Ergod. Th. \& Dynam. Sys. 1 (1981), 1-7.

[3] P. Gabriel. Sur la structure des extensions à deux points du produit d'une transformation par un schéma de Bernoulli. To appear.

[4] M. R. Herman. Construction d'un difféomorphisme minimal d'entropie topologique non nulle. Ergod. Th. \& Dynam. Sys. 1 (1981), 65-76.

[5] D. Ornstein. Ergodic Theory, Randomness and Dynamical Systems. Yale Math., 1974.

[6] D. Ruelle. Ergodic theory of differentiable dynamical systems. Publ. de l'IHES 50 (1980), 27-58.

[7] J. P. Thouvenot. Quelques propriétés des systèmes dynamiques qui se décomposent en un produit de deux systèmes dont l'un est un schéma de Bernoulli. Israel J. Math. 21 (1975), 177-207.

[8] J. P. Thouvenot. Remarques sur les systèmes dynamiques donnés avec plusieurs facteurs. Israel $J$. Math. 21 (1975), 215-232. 